\title{
Therapeutic Effect of Tetrax based Visual Biofeedback Training for Balance Dysfunction in a Child with Ataxic Cerebral Palsy- A Case Report
}

\author{
Somya Prasad \\ Consultant Occupational Therapist, VIMHANS Hospital, 1 Institutional area, Nehru Nagar, New Delhi, India. \\ Goodricke Group Hospital, Aibheel Tea Garden, P.O. Chalsa, Dist. Jalpaiguri 735206 (West Bengal)
}

\begin{abstract}
Background and Purpose: The study investigated the effectiveness of Tetrax biofeedback training on balance of a child with ataxic Cerebral Palsy (ACP). Both Tetrax and conventional Occupational Therapy (COT) were performed. Each session of Tetrax and COT was carried out for 30 minutes, 3 times per week for 4 weeks. Primary outcome measure was Berg balance Scale (BBS) and fall index (FI). Secondary outcome measures were the stability index (ST) and the weight distribution index (WDI). Tetrax training has effectively aided in the recovery of balance when used in addition to the COT treatment, however further studies with larger populations are required for evidence of its effectiveness.
\end{abstract}

Keywords: Ataxia, Tetrax, Balance, Biofeedback, Rehabilitation

\section{INTRODUCTION}

Cerebral Palsy (CP) is defined as a developmental disability resulting from brain injury that occurs before cerebral development is complete, characterized by delayed motor milestones and impaired motor control ${ }^{1,2,3}$. Birth complications like asphyxia are estimated to account for about six percent of patients with congenital $\mathrm{CP}^{1}$. Ataxic $\mathrm{CP}$ is the rarest form of $\mathrm{CP}$ and affects five to ten percent of patients and is predominately characterized by the presence of ataxia, hypotonia, dysmetria and a widebased gait ${ }^{1,3}$.

The most important contributing factor to difficulty with gait and reaching movement is poor balance control/ postural control. Reactive balance control is the ability of a person to recover from an unexpected balance disturbance due to slips or trips. Balance/ Postural Control is assessed by means of posturography, which is the quantitative analysis of centre-of- pressure (COP) as measured by force plates $^{2,4,5}$.

Due to their low-cost, video games that are classified as nonimmersive virtual reality systems, are the most used equipment in rehabilitation programs ${ }^{3,8}$. These video games are designed with the concepts of neuroplasticity ${ }^{8}$.

Research has indicated that impaired balance has a correlation with functional limitations in children with CP. Hence, therapy programs have increase in postural function as one of their goals with the aim of creating increased efficiency in both undisturbed stance and reactive balance control $^{2,4}$. The use of COP-controlled, game-based exercise protocols have been reported to improve subjects' dynamic balance control ${ }^{8}$. The Tetrax has been used to measure postural stability in several previous studies ${ }^{7}$.Thus, considering the use of posturography with neurological patients, the aim of this study was to evaluate the effectiveness of Tetrax Posturography on 
static, dynamic and gait balance of a child diagnosed with Ataxic CP.

\section{METHODOLOGY}

This is a prospective, longitudinal, and descriptive type case study authorized by the child's parents by signing an informed consent.

\section{CASE DESCRIPTION}

\section{History}

A 10-year-old male child, diagnosed with clinical ataxic CP was included in the study. The child had cognitive preservation and independent gait without auxiliary devices, albeit with difficulty in running, jumping, climbing and descending stairs. The child had no history of previous respiratory, cardiac or orthopedic diseases and received Occupational Therapy services at a tertiary hospital in New Delhi, India.

\section{Instrumentation}

Tetrax Interactive Static Posturography System (Tetrax $®)$ [Sunlight Medical Ltd, Israel] is a COP-controlled, video game-based exercise system designed for patients with either orthopedic or neurological problems ${ }^{8}$. It is based on the measurement and computerized elaboration of electronic signals emitted by four-foot plates, one for each heel and toe. A tetrax training program is known to improve standing stability. The Tetrax is also known as an effective balance-status measurement instrument ${ }^{9}$. Measurements are made in eight different conditions and each position takes about 40 seconds: (i) head straight, eyes open, on hard ground (NO); (ii) head straight, eyes closed, on hard ground (NC); (iii) head straight, eyes open, on soft ground (foam under feet) [PO]; (iv) head straight, eyes closed, on soft ground (PC); (v) head turned to the right, eyes closed (HR); (vi) head turned to the left, eyes closed (HL); (vii) head backwards $30^{\circ}$, eyes closed (HB); and (viii) head forward $30^{\circ}$, eyes closed (HF).

Vestibular, visual, and somatosensorial inputs are recorded in each of the eight different positions to evaluate static postural balance and fall risk ${ }^{7}$.

\section{Intervention}

The subject received conventional Occupational Therapy as baseline for 40 minutes in an outpatient setting where the subject worked on gait, balance, and motor coordination. He also received Tetrax biofeedback balance training for 20 minutes for 3 days a week for 4 weeks in the same outpatient setting, totaling 12 sessions.

During the Tetrax sessions, the following eight games were used (Figure 1): 'Skyball' (left-right weight shifting, improving reaction time, tracking a moving object, attention focus); 'Tag' (anteriorposterior weight shifting, improving reaction time, tracking a moving object, attention focus); 'Immobilizer' (static symmetric weight bearing, Weight distribution, weight transfer); 'Target' (multidirectional weight shifting, combining correct balance with cognitive tasks); 'Gotcha' (left-right weight shifting, multidirectional weight shifting, improving reaction time, tracking a moving object, attention focus); 'Speedball' (anteriorposterior weight shifting, improving reaction time, tracking a moving object, attention focus); 'Freeze' (full weight bearing of the limb); 'Catch' (weight distribution, tracking a moving object, weight transfer, attention focus) ${ }^{8}$.

The level challenge was adjusted by target size, number of targets appearing on the screen at the same time, and speed of target movement. The challenge progression was tailored according to the subjects' ability. At the end of each game, the final score and percentage of corrected hits on the left and/or right side are shown on the screen to give objective feedback about the subject's performance.

The treating therapist chose 3-5 games for each intervention session. The treating therapist demonstrated how to stand on the force plate and how to move the dot on the screen from movements at the ankles without lifting the foot from the force plate 
Somya Prasad. Therapeutic effect of tetrax based visual biofeedback training for balance dysfunction in a child with ataxic cerebral palsy- a case report.

by leaning forward, backward and on the left and right side. The subject was then asked to try out. Before beginning, the therapist verified that the subject understood

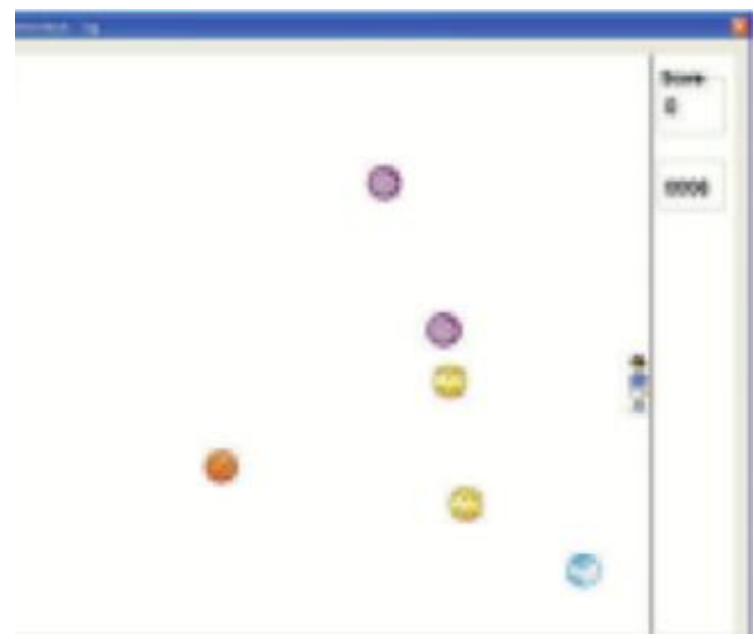

Skyball: Move the baseball glove to catch the baseballs by moving right-to- left.

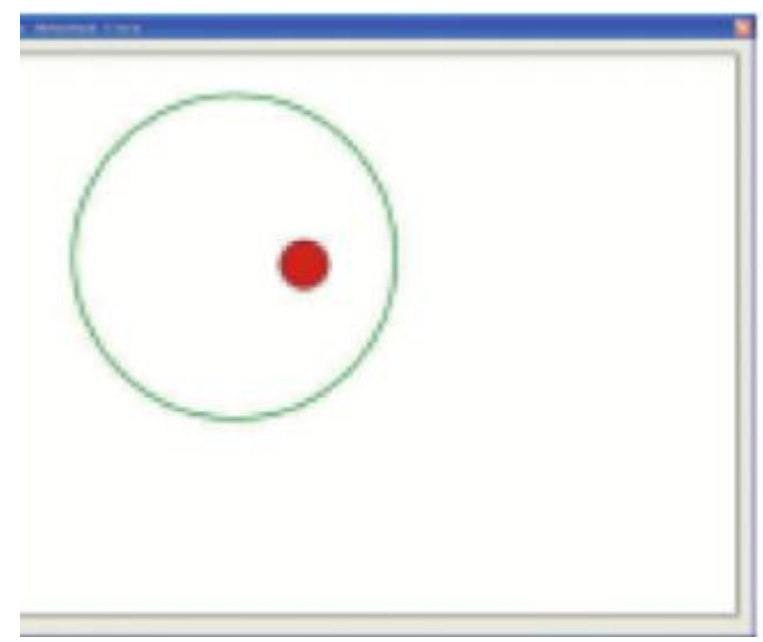

Immobilizer: Keep the top of each of the columns within the green section by keeping steady.

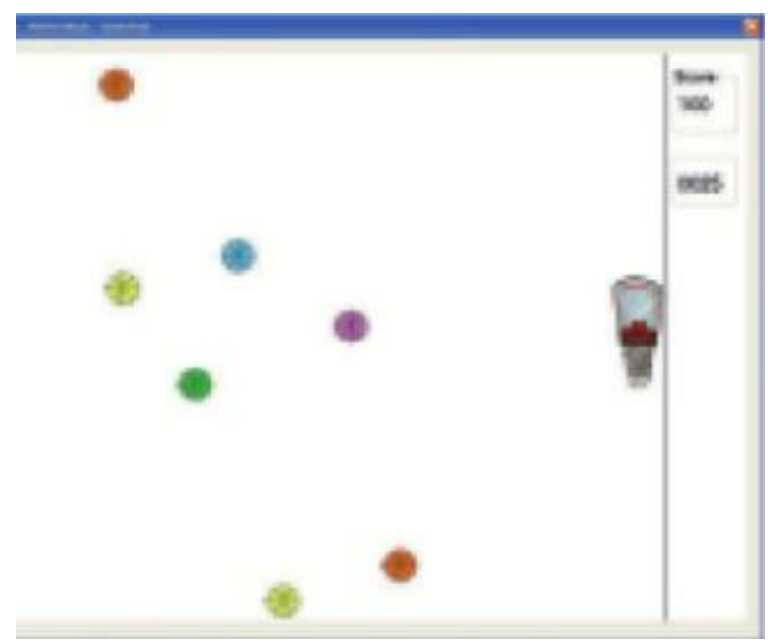

Target: Catch each of the targets in turn with the red ball. the functioning of the feedback system and that the subject had adopted a comfortable and suitable foot position.

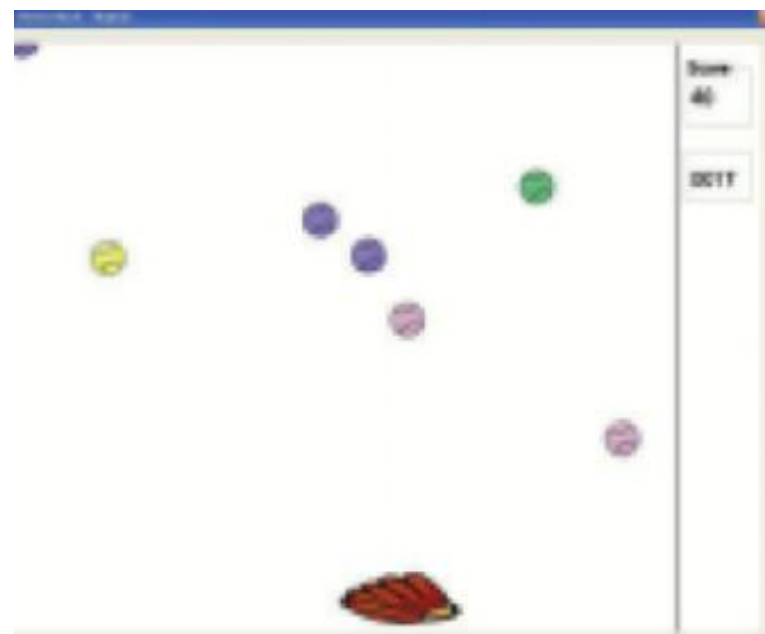

Tag: Move the soccer player to avoid the soccer balls by moving front-to- back.

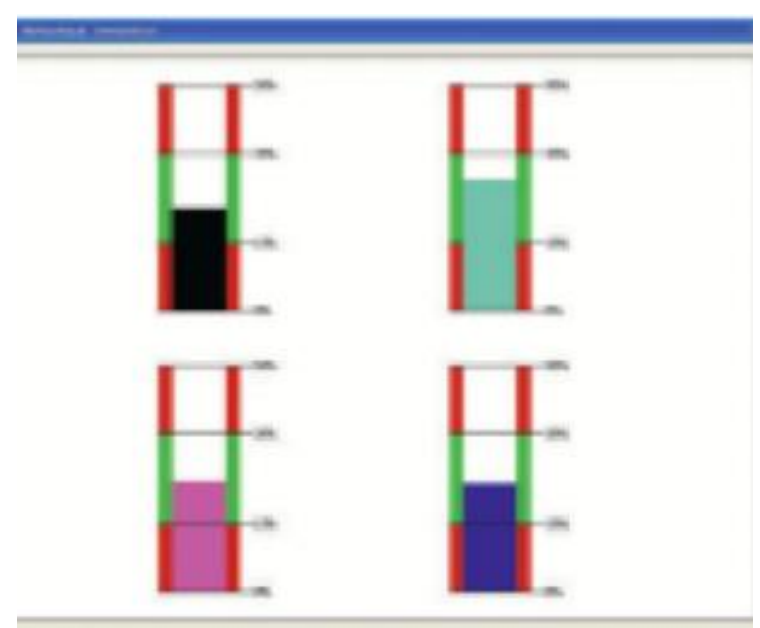

Freeze: Keep the red ball inside the circle by keeping steady.

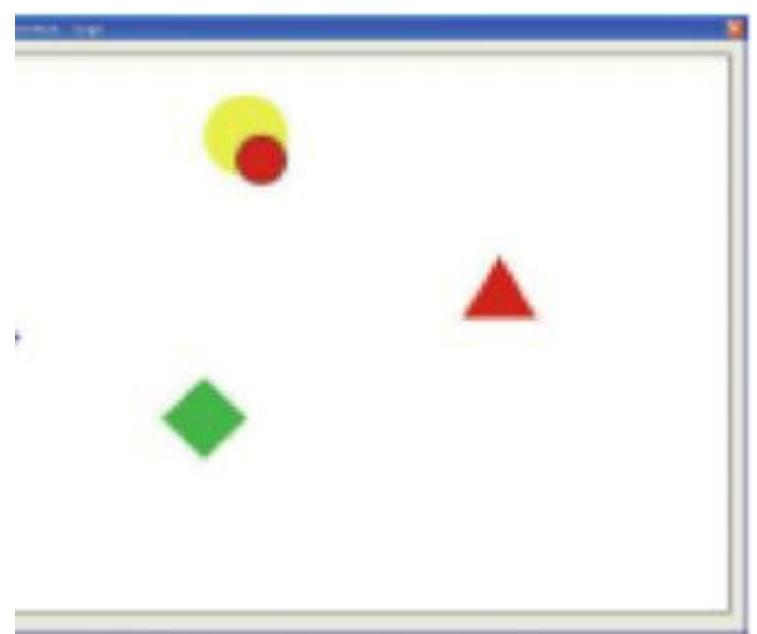

Speedball: Move the basketball hoop front-to-back to catch the Basketballs. 


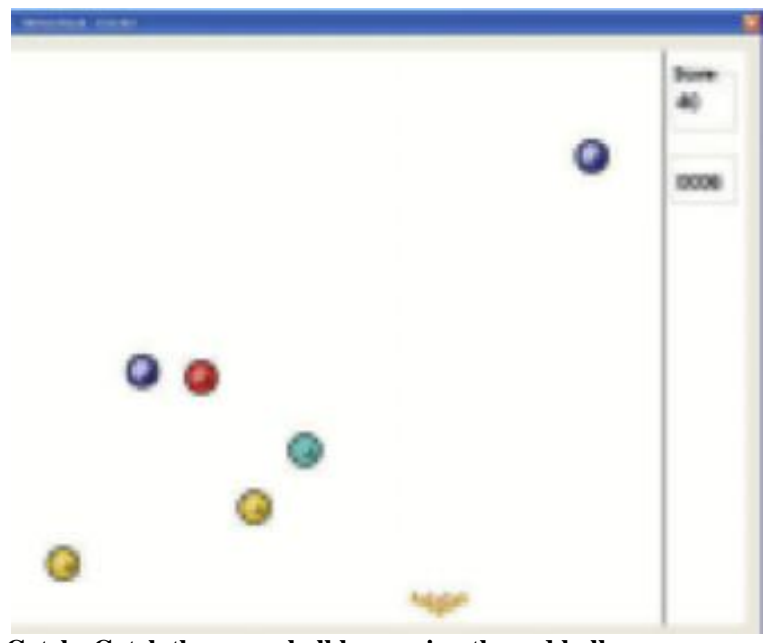

Catch: Catch the green ball by moving the red ball.

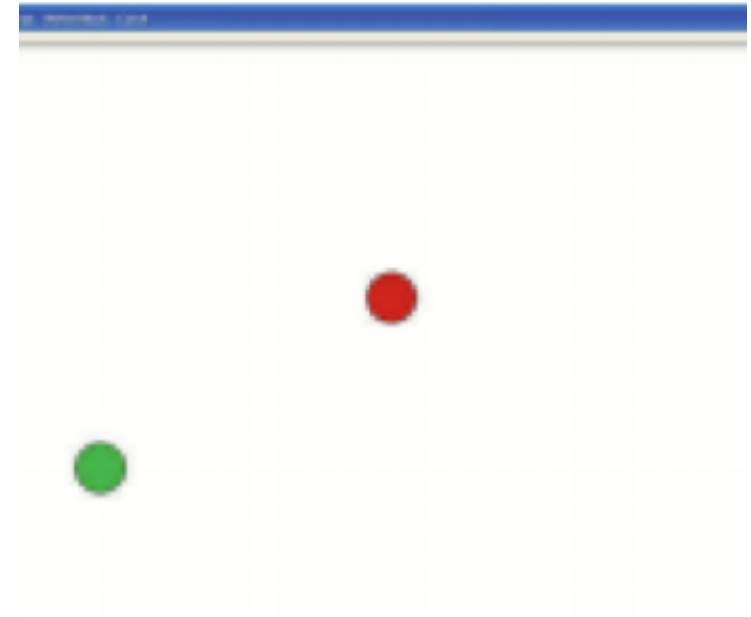

Gatcha! : Move the bowling pins right-to- left to avoid the bowling balls

Figure 1: The 8 Games played on Tetrax by the Subject

Table 1: Summary of Balance Activities as performed by the Subject

\begin{tabular}{|l|l|}
\hline Body Stability Tasks included: & Quiet Standing (with normal as well as narrow BOS*) \\
& Standing with eyes closed \\
& Tandem standing \\
& Kneeling on hard surface and soft mattress \\
\hline Body Stability and Manipulation Tasks included: & Standing with rapid alternating hand movement \\
& Standing on balance board and reaching in all directions \\
& Throwing and catching a ball while standing \\
\hline Body Transport Tasks included: & Walking with normal and narrow BOS \\
& Stair climbing \\
& Walking backward \\
& Walking sideways \\
& Walking on soft mattress \\
& Tandem walking on balance beam \\
\hline Body Transport and Manipulation Tasks included: & Walking while counting alternatively from 1-100 \\
& Walking while carrying books \\
& Walking in a busy hallway \\
\hline
\end{tabular}

Table 1 shows a summary of balance activities for the subject.

\section{Outcome Measures}

The subject was evaluated before and after the four weeks of training. The primary outcome measures were the Beg Balance Scale (BBS) to analyze static balance and Fall index (FI). The secondary outcome measures were the stability index (ST) and weight distribution index (WDI). The FI, ST and WDI scores were measured using the Tetrax instrument.

Higher ST and FI shows poorer postural control ${ }^{7,9,10}$. The normal weight distribution is $25 \%$ on each plate. If a patient's weight distribution changes, it increases the WDI and shows a poorer postural control ${ }^{9,10}$.

\section{RESULTS}

Table 2 shows the data regarding the change in static balance as measured by the BBS. There was an increase in the total score from 31 to 45 , with most of the progress in items that require standing unsupported with both feet together and eyes closed, reaching forward with outstretched arm in standing, transfers, looking back over the shoulder in standing, turning $360^{\circ}$, alternating feet on a step, standing with one foot in front of the other and standing on one leg.

When assessing his balance via Tetrax, the Fall index shows the degree of risk of falling on a scale of 0-100: low risk 0-35, moderate risk 36-57, high risk 58-100 11. The subjects' FI was in high risk both pre and post training with scores being 100 and 89. Pre-intervention WDI score was 7.74 

with ataxic cerebral palsy- a case report.

and ST was 84.44 in eyes-open and 5.11 and 151.46 in eyes-closed respectively. Postintervention WDI scores were 6.14 in eyes- open and 3.62 in eyes-closed. Postintervention ST scores were 74.63 in eyesopen and 124.07 in eyes- closed.

Table 2: BBS Scores pre- and post- treatment.

\begin{tabular}{|l|l|l|}
\hline Balance Item & Pre-treatment Score & Post-treatment Score \\
\hline Sitting to Standing & 3 & 4 \\
\hline Standing 2 minute Unsupported & 4 & 4 \\
\hline Sitting Unsupported & 4 & 4 \\
\hline Standing to Sitting & 3 & 4 \\
\hline Transfers & 3 & 4 \\
\hline Standing Unsupported with Eyes Closed & 2 & 4 \\
\hline Standing Unsupported with Feet Together & 0 & 2 \\
\hline Reaching Forward & 2 & 3 \\
\hline Pick an object from the floor & 3 & 3 \\
\hline Looking back over the shoulder & 3 \\
\hline Turn 360 & 2 & 3 \\
\hline Alternating feet on a step and standing & 2 & 3 \\
\hline Standing, with one foot in front of the other without support & 0 & 1 \\
\hline Standing on one leg & 2 & 3 \\
\hline TOTAL SCORE & 31 & 45 \\
\hline IMPROVEMENT PERCENTAGE & $25 \%$ \\
\hline \multicolumn{2}{|c|}{ Data in absolute values and percentage of total gain. } \\
\hline
\end{tabular}

Figure 2 shows a plot of subjects' COP at baseline (pre-intervention) and at postintervention. The decrease in postural sway is clearly visible in the plot also the COP is closer to the midline in the post-intervention session then at baseline.

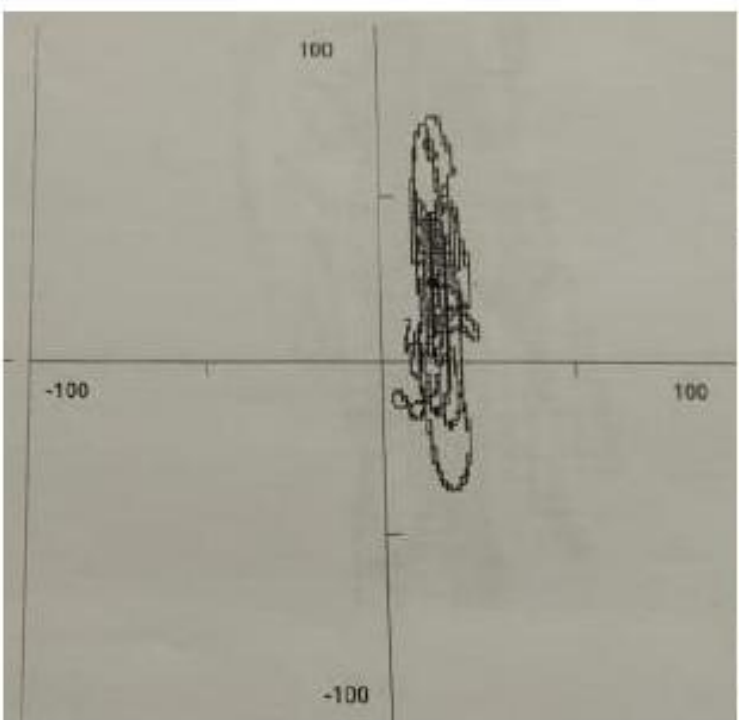

(a)

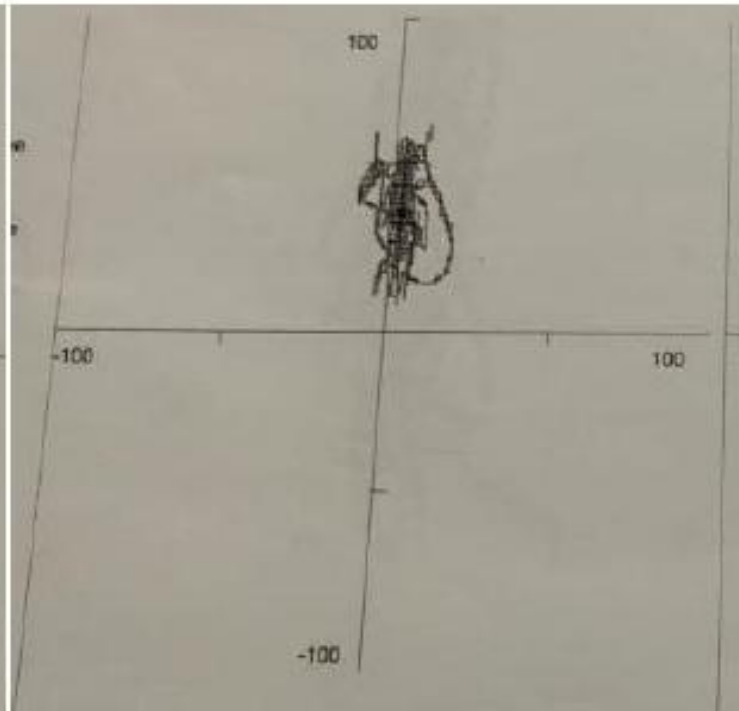

(b)

Figure 2: A plot of Center of Pressure at (a) Baseline (Pre-treatment), and (b) Post-treatment

\section{DISCUSSION}

Ataxic CP children are characterized by the presence of hypotonia and dysmetria, hindering their balance and movement coordination $^{3}$. Rehabilitation for these children is a long-term commitment, which becomes monotonous and tiresome. Including Tetrax as a treatment modality along with conventional therapy can provide several benefits, which have been already proven by scientific studies ${ }^{6,7,8}$.

After tetrax training, this study showed an increase in the BBS scores, specifically in the items that require standing unsupported with feet together, standing on one leg, climbing stairs using alternative feet without the support of upper limbs, standing unsupported with eyes closed, looking back over the shoulders and 
forward reach while standing. This was probably a result of the games that the subject played in Tetrax which targeted characteristics such as motor coordination, weight bearing and weight displacement, forward and backward sway, postural adjustments, and balance in a more interactive and playful manner for the subject.

Providing $\mathrm{CP}$ children with simultaneous visual feedback about postural sway may help in creating a more external focus of attention that is the postural sway becomes apparent via the visual cursor movements. This visual feedback of postural sway provides a functional perspective for postural control which in turn improves the performance of the primary $\operatorname{task}^{5}$.In the tetrax program, the patient stands on four fixed flat force plates. The COP can be seen by the patient on the screen, and patients can achieve the required tasks by moving the COP. Patients move their COP voluntarily, but within the limited range of the Tetrax instrument. Also, their feet were required to be always placed on the force plate to accomplish the task. Hence, the beneficial effects of Tetrax may be due to stimulation of the static balance control $^{9}$. Accordingly, the SI and WDI scores were improved in both open and closed-eyed positions.

Other studies have also proved that using tetrax provides functional improvement in neurological patients. According to Hung et $\mathrm{al}^{8}{ }^{\mathrm{B}}$, chronic Hemiplegic patients showed improvements in reaction time, proprioception and symmetric weight bearing, gait, and forward reach after rehabilitation with Tetrax ${ }^{8}$. In another study performed by Ledebt et $\mathrm{al}^{6}$ on patients with hemiplegic $\mathrm{CP}$ by the visual feedback training using the force plates over 18 sessions, there was a general improvement observed for stance and gait ${ }^{6}$.

The study had a few limitations. (i) Small sample size; (ii) The duration of the intervention was short (20 minutes, 3 days a week for 4 weeks), may have undervalued the effects of the intervention; (iii) lack of follow-up assessment leading to no documentation of carryover effects of the Tetrax biofeedback system.

The results in this case study are not powered to make any definite conclusion about the efficacy of the Tetrax biofeedback training. Repeating the study in larger and heterogenous group of patients with a longer period of intervention will provide more detailed information about fall risk in different age groups and in specific disorders and is needed to explore the effects of this intervention. Also, assessing the long-term effects of Tetrax biofeedback training is also suggested.

\section{CONCLUSION}

Using Tetrax during treatment of an Ataxic CP child can be of beneficial nature, this is because the ST and WDI scores improved in both open-eyed and closedeyed positions. A Tetrax program may benefit patients with abnormal proprioceptive function. However, further studies to provide more robust data about the efficacy of this intervention is warranted.

\section{Acknowledgement: None}

\section{Conflict of Interest: None}

\section{Source of Funding: None}

\section{REFERENCES}

1. Krigger KW. Cerebral Palsy: An Overview [Internet]. 2006; 73(1). Available from http://www.aafp.org/afp

2. Shumway-Cook A, Hutchinson S, Kartin D, Price R, Woollacott M. Effect of balance training on recovery of stability in children with cerebral palsy. Developmental Medicine and Child Neurology. 2003; 45: 591-602

3. Ribeiro da Silva R, Iwabe-Marchese C. Using virtual reality for motor rehabilitation in a child with ataxic cerebral palsy: case report. Fisioter Pesq. 2015; 22(1):97-102

4. Wollacott MH, Shumway-Cook A. Postural Dysfunction During Standing and Walking in Children with Cerebral Palsy: What are 
the Underlying Problems and What New Therapies Might improve Balance? Neural Plasticity. 2005; 12(2-3): 211-219

5. Donker SF, Ledebt A, Roerdink M, Savelsbergh GJP, Beek PJ. Children with cerebral palsy exhibit greater and more regular postural sway than typically developing children. Exp Brain Res. 2008; 184:363-370

6. Ledebt A, Becher J, Kapper J, Rozendaal RM, Bakker R, Leenders IC, Savelsbergh GJP. Balance Training with Visual Feedback in Children with Hemiplegic Cerebral Palsy: Effect on Stance and Gait. Motor Control. 2005; 9: 459-468

7. Akkaya N, Donganlar N, Celik E, Engin AS, Akkaya S, et al. Test-Retest Reliability of Tetrax Static Posturography System in Young Adults with Low Physical Activity Level. Int J Sports Physical Therapy. 2015; 10(6):893-900

8. Hung J, Yu M, Chang K, Lee H, Hsieh Y, Chen P. Feasibility of Using Tetrax Biofeedback Video Games for Balance Training in Patients with Chronic
Hemiplegic Stroke. American Academy of Physical Medicine and Rehabilitation. 2016; $1-9$

9. Song, Chun, Kim, Lee SJ, Yi JH, Park DH. The Effect of Virtual Reality and TetraAtaxiometric Posturography Programs on Stroke Patients with impaired Standing Balance. Annals of Rehabilitation Medicine. 2014; 38 (2): 160-166

10. Kim H, Yun DH, Yoo SD, Kim DH, Jeong YS, Yun J et al. Balance Control and Knee Osteoarthritis Severity. Annals of Rehabilitation Medicine. 2011; 35:701-709

11. Shin S, An D. The Effect of Motor Dualtask Balance Training on Balance and Gait of Elderly Women. J. Phys.Ther. Sci. 2014;26:359-361

How to cite this article: Prasad S. Therapeutic effect of tetrax based visual biofeedback training for balance dysfunction in a child with ataxic cerebral palsy- a case report. Int $J$ Health Sci Res. 2021; 11(9): 101-107. DOI: https://doi. org/10.52403/ijhsr.20210915 